Research Article

\title{
Development of a Monitoring and Management System for Nonheritage Tourist Attractions Based on Mobile GIS and Multisensor Technology
}

\author{
Enhou Zu, ${ }^{1}$ MingHung Shu $\mathbb{D},{ }^{2,3}$ JuiChan Huang $\mathbb{D},{ }^{4}$ TzuJung Wu, ${ }^{5}$ ChihWei Hsu $\mathbb{D}^{2}{ }^{2}$ \\ and YuanChieh Chang ${ }^{5}$ \\ ${ }^{1}$ School of Management, Henan University of Science \& Technology, Kaiyuan Avenue, Luoyang, Henan 471023, China \\ ${ }^{2}$ Department of Industrial Engineering and Management, National Kaohsiung University of Science and Technology, \\ Kaohsiung City 807618, Taiwan \\ ${ }^{3}$ Department of Healthcare Administration and Medical Informatics, Kaohsiung Medical University, \\ Kaohsiung City 80708, Taiwan \\ ${ }^{4}$ Yango University, Fuzhou, Fujian 350015, China \\ ${ }^{5}$ Institute of Technology Management, National Tsing Hua University, Hsinchu, Taiwan
}

Correspondence should be addressed to ChihWei Hsu; i108143102@nkust.edu.tw

Received 19 May 2021; Revised 16 June 2021; Accepted 10 July 2021; Published 22 July 2021

Academic Editor: Sang-Bing Tsai

Copyright $\odot 2021$ Enhou Zu et al. This is an open access article distributed under the Creative Commons Attribution License, which permits unrestricted use, distribution, and reproduction in any medium, provided the original work is properly cited.

With the rapid development of the tourism industry, how to monitor scenic spots and tourists in real time has become an issue. This paper mainly describes the development of a monitoring and management system for scenic spots of intangible cultural heritage based on Mobile GIS and multisensor technology. The monitoring system adopts the idea of structured programming, which reduces the coupling degree of various components and promotes the expansion of system functions. The shortest path module uses the scenic spot management subsystem on the PC. The scenic spot manager enters the distance between adjacent scenic spots that can be directly reached into the system database and then uses the shortest path algorithm, Dijkstra algorithm, to calculate. Asynchronous Socket programming mechanisms are used to implement communication capabilities, the XML markup language is selected as the system's data exchange protocol, and the DHT11 digital temperature and humidity sensor is used to obtain humidity information around ancient buildings. A Mobile GIS reader of an ancient building in a scenic spot sends a request to connect to a server. The listener is the communication interface between the server software and the reader. It is responsible for parsing the transmitted data and storing it in the database. The CC2430 chip is used to wear on tourists. When tourist nodes and guide nodes enter the scenic spot, they join the network to query the density of the entire scenic spot and upload real-time information. In terminal query, the average response time of real-time location query is $2 S$. The average initial response time for historical location queries is about 3S. The results show that the visualization services provided by software development can intuitively and accurately display the flow and density of scenic spots, providing a scientific reference for carrying capacity and flow management of scenic spots.

\section{Introduction}

With the continuous changes in technology, especially the new generation of information technology centered on the Internet, cloud computing, and big data, it has become the driving force behind the rapid development and change of the world economy and has broadened the possibility of human society entering the era of intelligence. People not only work, study, and live in an intelligent environment but also use intelligent information technology to conduct scientific decision-making analysis, implement refined management, and achieve sustainable economic and social development. In recent years, the National Tourism Administration has attached great importance to the work of 
smart tourism, and various regions have recognized the work of smart tourism as an important issue and have consistently implemented a number of measures to strengthen this initiative. Therefore, it is very necessary to develop a scientific and sensible monitoring and management system for tourist attractions.

With the increasingly fierce competition in the tourism market, the contradiction between the backward industry management level and the rapidly developing tourism market is increasing. With the vicious competition in the tourism market, false quotation propaganda will produce the phenomenon of "no group fee." This will greatly undermine the economic balance between tourism companies and other related companies. The level of tourism services has decreased, the itinerary plan has shrunk, and the phenomenon of self-care items and shopping has been greatly increased. This series of behaviors infringing on the rights and interests of consumers, as a result, reduced tourists' desire to consume, and tourism resources suffered predatory consumption and devaluation. Tourism professionals have lost confidence in the industry and this caused great damage to the tourism market. Consumers urgently need a standardized market to safeguard and protect their own interests. Tourism professionals also need good discipline and means to curb competition. The tourism market needs a standardized management method to facilitate the display of graphic signs and publicity over the Internet. Travel companies publish fair tourism information to curb false advertisements, enhance industry management internally through high-tech information collection and management technology, and maintain a good pricing and procurement system to ensure the proper development of the tourism industry.

Li Yang faced the Android platform and used Java language, combined with GPS technology, to develop a Mobile GIS navigation service system, with the help of the Android smartphone operating system [1]. Dong Ying studied the key technologies of Mobile GIS such as map data loading and offline travel information storage and developed a travel service system that integrates travel map services, information query, and information sharing [2]. Tewari and Gupta proposed an ultra-lightweight two-way authentication protocol that only uses bitwise operations, so it is very effective in terms of storage and communication costs. In addition, the computational overhead is very low. They also compared the proposed work with existing work and studied brief cryptanalysis of the protocol to ensure nontraceability. Their research lacks practical practice [3]. Liu et al. believed that the use of blocking tags will make the classic Mobile GIS estimation problem more challenging, because some real tag IDs are covered by blocking tags, and some are not. They proposed the Mobile GIS estimation scheme with barrier tags (REB), which is the first Mobile GIS estimation scheme with barrier tags. REB uses the Aloha protocol with framed time slots specified in the EPC C1G2 standard. For each round of the Aloha protocol, REB first executes the protocol on the real tag and the blocker tag and then uses the same Aloha protocol parameters to virtually execute the protocol on the known blocker ID. The Mobile GIS estimation scheme they proposed has low reliability [4]. Shangguan et al. proposed a method called Mobile GIS-based relative object positioning (STPP). By moving the reader to a set of tags, during this period, the reader keeps interrogating the tags. For each tag, the reader obtains a series of RF phase values from the tag's response, by analyzing the spatiotemporal dynamics in the phase profile, STPP can calculate the spatial order between tags. The method they proposed is not accurate enough [5].

This research gives a brief overview of Mobile GIS technology, makes detailed research and analysis on the application of Mobile GIS technology in the management and service of tourist attractions, and achieves the application of intelligent electronic tickets for tourist attractions and tourist sightseeing services. The composition and working mechanism of the Mobile GIS system are briefly introduced. After a simple analysis of the technical specifications and standards of the entire Mobile GIS system, the specifications and selection of the Mobile GIS hardware used in this study are given, and the functional characteristics and functional characteristics of the selected hardware identification are listed. The technical parameters provide detection equipment to monitor the present dissertation, describe in detail the construction of the Mobile GIS event collection network for passengers and the model and method of processing the Mobile GIS tourist visit, and explain network technology, data transmission technology, and reader installation technology in the area. Based on the analysis of the semantics of the Mobile GIS event of passenger reading, the principles and methods of implementation of the statistics of calculation of passenger flow and probability distribution, the determination of tourist locations, and the following and traces of tourist routes are given.

\section{Monitoring and Management of Intangible Heritage Tourist Attractions}

2.1. Internet of Things. The Internet of Things (IoT) is an evolving architecture that connects multiple devices to the Internet to communicate or receive updates from the cloud or server. In the future, the number of these connected devices will greatly increase, making them an integral part of our daily lives. Although these devices make our lives more comfortable, they also put our personal information at risk. Therefore, the safety of these devices is also a major issue today $[6,7]$.

The application service layer of the Internet of Things includes two parts: the application system and the common support platform. This layer is related to the specific application of the industry. It is a specific application of the Internet of Things project. Common support platforms include cloud computing platforms, high-performance computing platforms, physical information exchange platforms, and other application resource platforms, and application systems include government application systems, enterprise application systems, and other specific application systems. At the application service layer, the combination of the Internet of Things technology and the professional technology of the industry to build a professional application 
system for the Internet of Things has an important impact on the national economy and social development [8].

2.2. Mobile GIS. Mobile GIS evolved based on DesktopGIS and WebGIS. Mobile GIS overcomes the unified work environment and poor mobility of DesktopGIS and WebGIS. Due to its portability, it has been used in various fields, especially on platforms. The application in the fields of construction, navigation, and location services is relatively rich, so it attracts domestic and foreign experts and designers to do a lot of research on it. The research on the construction of Mobile GIS platform is the basic work of Mobile GIS. Esri, Google, Baidu, Tiantu, and so on provide different solutions for different scenarios. Esri has been deeply involved in the GIS field for many years, leading the wave of Mobile GIS development, and provides ArcGISRuntimeSDK for Android/IOS for the application industry [9, 10]. For the overfrequency carrier $f$, let the transmitting phase be $\Phi$, and the transmitting signal and receiving signal of the reader can be expressed as

$$
\begin{aligned}
& s(t)=\left[\cos \left(2 \pi f_{0} t+\phi_{s}\right)+A\right] \cdot \cos \left(2 \pi f_{c} t+\phi_{c}\right), \\
& r(t)=\left[\cos \left(2 \pi f_{0} t+\phi_{r}\right)+A\right] \cdot \cos \left(2 \pi f_{c} t+\phi_{d}\right) .
\end{aligned}
$$

Among them, $\phi_{d}=\phi_{c}+f_{c} \cdot \tau_{d}$. With the increasing popularity of radio frequency identification (RFID) technology in the retail and logistics industries, RFID privacy issues have aroused great attention, because tags will respond to information queries from users regardless of whether the responder is authorized or not [11]. Let $\phi_{r}=\phi_{s}+f_{0} \cdot \tau_{d}$; then

$$
\begin{aligned}
& s(n)=\left[\cos \left(\frac{2 \pi n f_{0}}{f_{s}+\phi_{s}}\right)+A\right] \cdot \cos \left(\frac{2 \pi n f_{c}}{f_{s}+\phi_{c}}\right), \\
& r(n)=\left[\cos \left(\frac{2 \pi n f_{0}}{f_{s}+\phi_{r}}\right)+A\right] \cdot \cos \left(\frac{2 \pi n f_{c}}{f_{s}+\phi_{d}}\right) .
\end{aligned}
$$

Among them, $f_{s}$ is the sampling frequency. In the actual application of Mobile GIS, the label attached to the item only identifies the item or stores a small amount of basic information. It is difficult for the system to understand the information changes between it and the environment through the tag, and it has its own transmission distance limit $[12,13]$. The advantages of WSN perceiving external information and networking can just make up for its complement, and it can undoubtedly increase its application range and enhance its functions [14].

2.3. WSN. A large number of wireless nodes in the Wireless Sensor Network (WSN) collect and transmit sensing data from the WSN coverage area in a cooperative manner and provide corresponding basic services for various application requirements $[15,16]$. As one of the current hot research directions, WSN has broad application prospects $[17,18]$.

(1) The sensor operates with low power consumption. Without external power support, wireless sensor nodes can operate independently for long periods of time. If they do not control their own power consumption, the nodes will quickly stop working and cause network paralysis. Thanks to the rapid development of micrometer and nanotechnology, microprocessing mechanical parts, microsensors, microactuators, high-performance lithium batteries, radio frequency modules, and signal processing modules can be integrated into a small chip, enabling current wireless sensors capable of operating at very low power. In an ideal free space, when the wireless signal is omnidirectional, its intensity decays squarely with the distance $[19,20]$.

$$
\mathrm{PL}(d B)=10 \lg \left(\frac{P_{0}}{P}\right)=10 \lg (L)+\Delta L,
$$

where $P$ is the signal received power [21].

(2) Random deployment emphasized network topology. Due to the limitation of the working environment, some WSN nodes are randomly scattered in the work area, the communication distance of the nodes is limited, and the node data needs to be sent and forwarded frequently. In order to ensure that the WSN can work continuously for a long time, the sensor nodes are in most of the time. Sleep state requires scheduling other active sensor nodes to take over the work of sleeping nodes. Redundant and dense node deployment can maintain enough active nodes to avoid premature paralysis of WSN $[22,23]$.

(3) Strengthen the self-organizing network between multiple nodes in the networking function. Due to the lack of fixed access points, just like the wireless ad hoc network, the communication between WSN nodes depends on mutual cooperation. The difference is that the hardware structure of sensor nodes is simple. In order to be able to adapt to work in a longterm and unmanned environment, WSN focuses more on node scheduling in self-organization and coordination. In addition to being able to easily join the network, it can also be able to autonomously change the routing configuration to meet the WSN's need for a compromise between energy consumption and performance [24].

2.4. Shortest Path Algorithm. The self-service tour guide part of the system realizes the shortest path planning function. The shortest path is a classic problem in graph search. In order to find the shortest path in the graph, many algorithms have been proposed to solve this problem. These algorithms have to achieve two goals: one is to finally find a path with the lowest cost, and the other is to optimize the algorithm as much as possible, that is, to take up a small amount of memory and find the shortest path as quickly as possible, which is to reduce the time complexity of the algorithm and space complexity $[25,26]$. Suppose the coordinates of the target node are $(x, y)$; then

$$
d_{n}^{2}=\left(x_{n}-x\right)^{2}+\left(y_{n}-y\right)^{2},
$$


where $d_{n}$ is the corresponding ranging value of each anchor node [27].

The most common problem with the shortest path is finding the shortest path between two specific nodes in the graph. The most classic algorithm used to solve this problem is the algorithm proposed by Dijkstra, which is the shortest path algorithm for determining the starting point; that is, it can create the shortest path from the starting point to all nodes in graph. After the Dijkstra algorithm was proposed, many generations of people made improvements to these implementation methods, but all were developed based on the Dijkstra algorithm. In the self-guided tour system, if tourists need to take the shortest route from place A to place $B$ for a tour, they most want to know which of the many paths from place A to place B is the shortest. The graph is composed of nodes and paths. The shortest path algorithm problem is a classic algorithm problem in graph theory research [28].

\section{Monitoring and Management Experiment of Intangible Cultural Heritage Tourist Attractions}

3.1. Software Overall Module Design. The hardware driver in the Mobile GIS detection graphic node is written according to that hardware, which mainly includes the master controller initialization, the low-frequency alarm driver, and the sensor driver. This system uses the structured programming idea to compile independent driver programs for each hardware unit, which reduces the link between each component and facilitates the expansion of system operation. Hardware drivers mainly include the following:

Main control module: The main control module is the core processing unit at the bottom of the system. The main initialization content includes configuration of crystal oscillator source settings and pin initialization.

Low-frequency wake-up module: The low-frequency wake-up module driver mainly includes the driver of the low-frequency wake-up sending module and the driver of the low-frequency wake-up receiving module.

Sensor module driver: The data input and output of the temperature sensor are completed by a single data pin; the humidity sensor uses a single bus protocol to realize data transmission; configuration parameters are read; ancient building abnormal state information is read.

GPRS module driver: The main controller of the ancient building Mobile GIS reader sends commands through the serial port to control the GPRS module. There are four different AT command types, and different commands are used to perform corresponding operations, such as setting port number, establishing TCP connection, and sending data.

GPRS module driver: The main controller of the scenic spot Mobile GIS reader sends commands through the serial port to control the GPRS module. Among them, there are four different AT command types. Different commands are used for corresponding operations, such as setting port numbers, establishing TCP connections, and sending data. Figure 1 shows the software framework of the Mobile GIS sensing node of ancient buildings in the scenic spot.

3.2. Shortest Path Module Design. The shortest path module passes through the scenic area management subsystem on the PC. The scenic area managers enter the distance between the directly reachable adjacent scenic spots in the scenic area into the system database. Then use the Dijkstra algorithm to calculate the shortest optimal path, and finally present it in the form of an image.

3.3. Communication Module Design. The transport layer protocols mainly include TCP and UDP. The former is connection-oriented and provides reliable data transmission services. The communication software part of this system is mainly realized by TCP connection. The server is the server, and the reader is the client. The client's Socket makes a connection request and requests to connect to the server's Socket. After the server responds to the client's Socket request, it establishes a thread and then sends the description of the server to the client. After waiting for the client to confirm, the connection is established, and the two parties can communicate data. The server can send command frames to the perception nodes of various parts of the ancient building through the reader to obtain basic data. The core of the TCP server is the network communication program, which needs to handle multiple Mobile GIS reader connections at the same time. There are two types of TCP communication: synchronous and asynchronous. However, the synchronous TCP communication mechanism will cause blocking, and it is not real time and can meet the requirements of the system. Therefore, this research uses the asynchronous Socket programming mechanism to realize the communication function.

3.4. Data Exchange Protocol Design. Choose XML markup language as the data exchange protocol of the system. Realtime message format definition: the communication platform and each subsystem are mainly based on XML file exchange, and real-time data exchange is supplemented by data exchange mode. Real-time data transmission functions are reserved, mainly to facilitate the transmission of control commands in the system in the future, and transmission.

3.5. Server Software Design. The primary function of the server software is to communicate between the Mobile GIS reader and the system database in the perception layer of the system, including communication and storage. During the data communication process, the Mobile GIS reader of the ancient building in the scenic spot sends a request to communicate with the server, and the listening program is the communication interface between the server-side software and the reader. It is responsible for analyzing the transmitted data and storing it in the database. Network data 


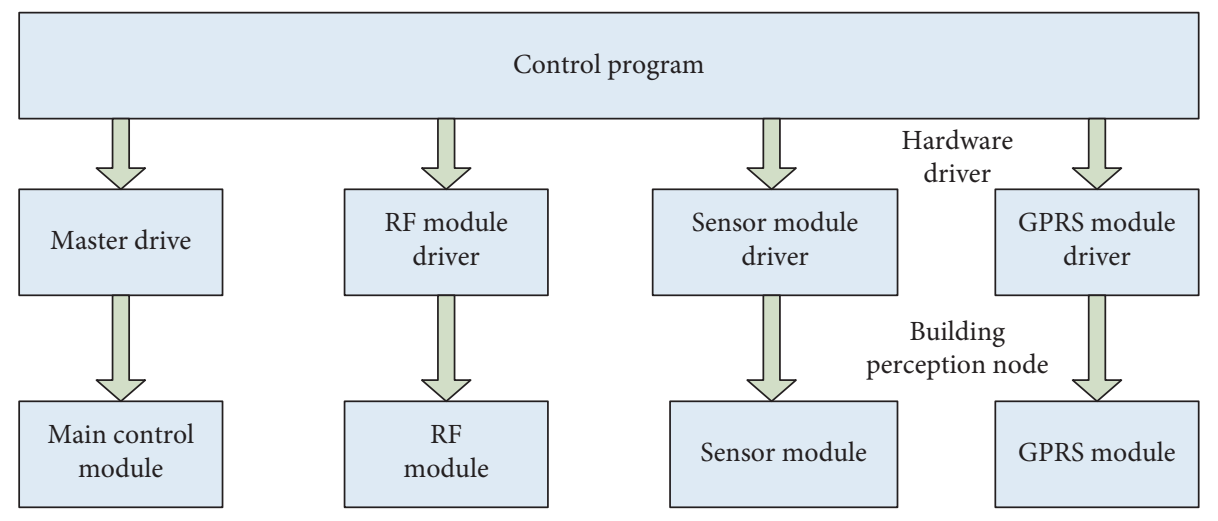

FIGURE 1: Mobile GIS sensing node software framework of ancient buildings in scenic spots.

communication is implemented using TCPSocket, which mainly considers the reliability of data transmission. After the data storage module is implemented by the communication software, it is necessary to design a set of reasonable and standardized data table structure to store the monitoring data of the intangible heritage scenic spot.

3.6. Scenic Network Layout. A wireless communication module is placed in the center of each cell, which is defined as a cell node. This module can communicate with other nodes entering the cell under its control, as well as with neighboring cell nodes. In this way, the network layout of the entire scenic spot is completed.

3.7. Design of Tourist Positioning Module. ZigBee wireless communication technology is applied to the Wireless Sensor Network to realize the positioning of tourists in the scenic spot. After the layout of the scenic spot network is completed, each cell node is fixed and known. After the cell node joins the network, it will be assigned a unique network: address and number, so as long as you know the network address or cell node number, you will know the location of the scenic spot. The CC2430 chip is used to wear on tourists. When the tourist nodes and tour guide nodes enter the scenic spot, they join the network, and the corresponding network addresses and numbers are also assigned. When the tour guide wants to know the location of a tourist at this time, the tour guide node sends a broadcast, which is forwarded by the community node until it is transmitted to the tourist node. The tourist node responds, and the response message returns in the reverse direction according to the path from the source node to the target node, containing the number of the cell nodes of the cell where the tour guide is located. After entering the time and tag ID, data collection starts. Most of the data collection needs to be carried out in some existing ideal environments. In this study, we assume that each unknown node can be connected to three anchor nodes, collect data, and deploy $n$ sensors in the established WSN environment. The node, the unknown node, is placed in it, the unknown node will communicate with the anchor node, and the cycle checks whether the number of communications with an anchor node is greater than or equal to three. If the above conditions are met, it means that the anchor node is connected to the unknown node, and the total number is increased by 1 . Otherwise, continue to collect the transmission information of the unknown node and the anchor node until the anchor node that meets the condition appears. Next, check whether the number of anchor nodes connected to the unknown node exceeds three. If it exceeds three, use RSSI value for weighted positioning, and use error correction to get the final coordinate position; otherwise continue to loop detection until the condition is met.

3.8. Humidity Monitoring. Temperature and humidity have an extremely important effect on the ancient buildings in the scenic area. Too high or too low temperature and humidity will cause irreversible damage to the ancient buildings in the scenic area. Therefore, it is extremely necessary to monitor the temperature and humidity of the ancient buildings. This research uses DHT11 digital temperature and humidity sensor to obtain humidity information around ancient buildings. DHT11's working voltage is $3-5.5 \mathrm{VDC}$, and it communicates with the main control chip through a singlewire serial interface, which has a very fast corresponding speed and accurate calibration ability. By adding a decoupling capacitor with a value of $100 \mathrm{nF}$ to the power supply pin, the stability of the DHT11 sensor is greatly improved. The DHT pin description is shown in Table 1.

3.9. Temperature Monitoring. This research uses DS18B20 from Dallas Semiconductor as the temperature sensor. It has the characteristics of small size, wide applicable voltage, simple interface, and so on. The temperature measurement range is $-55^{\circ} \mathrm{C}$ to $+125^{\circ} \mathrm{C}$, the accuracy is $\pm 5 \%$, and only one IO is required. It can complete the communication and signal transmission with the main control module. The data pin of DS18B20 is connected with the main control chip.

3.10. Level Monitoring. Due to the long history of ancient buildings in the nonheritage scenic area and due to the influence of natural and human factors, ancient buildings are constantly undergoing small changes such as increase in storeys, displacement, uplift, decline, and tilt. This kind of external physical influence has a fatal impact on ancient 
TABLE 1: DHT pin description.

\begin{tabular}{lcc}
\hline Pin & Name & Comment \\
\hline 2 & VDD & Power supply 3-5.5 V DC \\
3 & DATA & Serial data; single bus \\
4 & NC & Empty feet \\
5 & GND & Please hang \\
\hline
\end{tabular}

buildings, and there are great potential safety hazards. Therefore, it is very important to monitor the tilted state of ancient buildings. In this study, a six-axis sensor MPU6050 launched by InvenSense was used to monitor the tilt of ancient buildings. MPU integration is very high; through the IIC interface, the complete 9-axis fusion calculation data is output to the application side; it comes with 1024-byte FIFO memory, which greatly reduces the system power consumption.

3.11. Function Test. Terminal real-time location information query test uses the handheld management terminal to submit real-time location query and judges whether the terminal real-time location information query function is implemented correctly according to whether the server correctly returns the query result and whether the terminal correctly receives the query result. Terminal historical location information query test: in this system, the historical location information query submitted by the terminal is a historical running track query. It uses the handheld management terminal to submit the trajectory query and judges whether the function is implemented correctly according to whether the server software correctly receives, parses, and obtains the corresponding query result and correctly returns and whether the terminal can correctly receive and display the query result.

\section{Monitoring and Management of Intangible Cultural Heritage Tourist Attractions}

4.1. Analysis of Tourist Positioning Module. The routing of the positioning system is simulated and analyzed according to the established positioning network simulation platform. Set 40 nodes here, a square area with a side length of $80 \mathrm{~m}$ is used as the simulation area, the interval between nodes is set to $50 \mathrm{~m}$, and the simulation time is set to 100 seconds. The media access control layer (MAC) uses the 802.15.4 protocol, and the communication distance between nodes is set to $15 \mathrm{~m}$. When the simulation is set to 20 seconds, the visitor is node 30 , the tour guide node enters the No. 1 cell, the tour guide is in the area of the cell node 1 , and the node 1 needs to send data to the node 30 . The data uses the CBR service source, the size of each packet is 70 bytes, and the maximum number of transmission packets is 10,000. Because the premise of the on-demand routing protocol is that all nodes have routing functions, the nodes in the simulation are all $\mathrm{RN}+$ nodes. To achieve the above simulation, you must write an Otcl script and use the code in the ZigBee networking process to set the code in the simulation scenario. The two are very similar and only need to modify the network parameters. Set up the ZigBee network; the Otcl script realizes the data transmission between the two nodes, relying on the node data transmission to start the on-demand route discovery process. According to the usage rules of the controls in NS2, start to create 2 UDP objects and associate the two corresponding to the source node and the target node; secondly, set corresponding values for their data attributes; finally, the time when the data start to be sent makes settings. The entire process of route discovery before data transmission can be analyzed through important events in the trace file. In order to see this process, the generated nam file will be used to observe. Through the visualization file, we observe that the 20th second record is shown in Table 2.

This record indicates that node 1 sent a route request command box to the network at 20,000,345,000 seconds. We found that the time the node receives this routing request command frame is slightly delayed relative to its sending time, because the node checks its own routing table after receiving the send command to determine if the routing table contains the destination node path. After the inspection, the route to the destination node was not found, the route discovery process begins, and the route request command box is transmitted over the network. The entry in the trace file indicates that the routing response command box will be reversed in the reverse direction according to the routing path of the routing request command box received from node 30. During its return process, the transit node will update the routing table according to this routing response command box. Node 1 finally received the routing response command frame in 21.882678616 seconds and the specified best routing path is 1-0-10-4-5. Based on this trail, it is judged that junction 5 is located near junction 4 . It is confirmed that tourists are in community No. 4.

\subsection{Performance Analysis of Dijkstra Algorithm.} Assuming that the working frequency of the radio frequency carrier is $915 \mathrm{MHZ}$ and the indoor environment is a rectangular space of $20 \mathrm{~m} \times 15 \mathrm{~m}$, the scenic environment is divided into $C=400 \times 300$ fine grids to facilitate the solution. The total number of reference tags $H=22$, and they are arranged in a triangular grid in the indoor environment. The side length of the triangular grid is $4.25 \mathrm{~m}$ and the bottom length is $4 \mathrm{~m}$. The convergence speed of the Dijkstra algorithm is tested by Monte Carlo simulation, where $I$ is the number of simulations, combined with the cumulative function to evaluate the algorithm performance and positioning accuracy. If Dijkstra's initial position is in the high incidence area where the target appears, its quality optimization radius is small. For example, the quality search radius of RT8 is $2.2081 \mathrm{~m}$, and the quality search radius of RT1 is $3.6170 \mathrm{~m}$. The performance analysis result of the Dijkstra algorithm is shown in Figure 2. It is easy to know from Figure 2 that the improved Dijkstra algorithm can quickly converge, and the minimum value of $F$ can be less than $0.6444 \mathrm{~m}$, while the global positioning corresponding to GA optimization is $0.8738 \mathrm{~m}$, and the positioning accuracy of the triangular grid distribution is $0.8667 \mathrm{~m}$. A certain number of reference tags are placed on the edge of the scenic 
TABLE 2: The 20th second record.

\begin{tabular}{|c|c|c|c|c|c|c|}
\hline Number of the tour guides & & Ro & ath add & & & Number of the tourists \\
\hline 1 & $0 \times 05$ & $0 \times 04$ & $0 \times 05$ & $0 \times 05$ & $0 \times 03$ & 1 \\
\hline 1 & $0 \times 05$ & $0 \times 04$ & $0 \times 02$ & $0 \times 05$ & $0 \times 03$ & 0 \\
\hline 2 & $0 \times 05$ & $0 \times 03$ & $0 \times 02$ & $0 \times 02$ & $0 \times 03$ & 10 \\
\hline 2 & $0 \times 05$ & $0 \times 03$ & $0 \times 02$ & $0 \times 02$ & $0 \times 01$ & 4 \\
\hline 3 & $0 \times 05$ & $0 \times 05$ & $0 \times 05$ & $0 \times 05$ & $0 \times 02$ & 5 \\
\hline
\end{tabular}

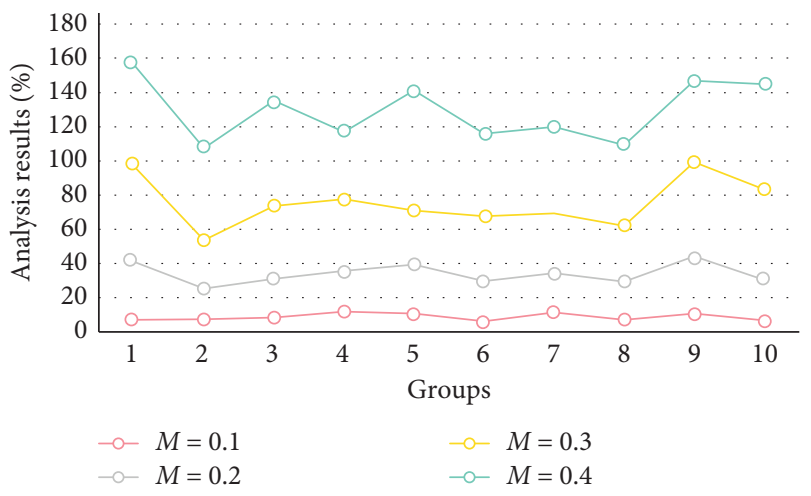

FIGURE 2: Dijkstra algorithm performance analysis results.

environment to cover the target area, such as tags RT1, RT2, and RT4; at the same time, a certain number of tags stay in areas with a higher probability of target occurrence, such as tags RT6, tags RT5, and tags R3, located around event A4. It needs to be pointed out that the Dijkstra algorithm in this chapter seeks to obtain the optimal deployment method of Dijkstra in the absence of noise. Therefore, this section uses 500 Monte Carlo experiments to further verify the global positioning accuracy and stability of the Dijkstra optimized deployment scheme under noisy conditions. When $M=0.3$, for the triangular grid-like uniform deployment method, $F$ is lower than $1.4492 \mathrm{~m}$ with a $50 \%$ probability, and the minimum value is also $1.3909 \mathrm{~m}$; for the deployment method obtained in this study, $F$ is lower than $1.3320 \mathrm{~m}$ with a $50 \%$ probability. Compared with the former, there is an increase of $8.09 \%$, while the maximum value of $F$ is controlled at $1.4024 \mathrm{~m}$. As $M$ increases from 1 to 3, the advantages of the optimized deployment method obtained in this study are gradually reduced compared to the triangular grid deployment method but still exist. The number of RREQ sent in the network before and after the algorithm is improved is compared and analyzed. We know that the node sending and receiving data will consume a certain amount of energy, and the energy consumed by sending and receiving data is much greater than the energy consumed when collecting and processing data. As can be seen from Figure 2, the number of RREQ messages sent in the improved algorithm is significantly reduced, which reduces the overall energy consumption of the network. The simulation results show that the improved algorithm is effective in controlling the transmission direction of RREQ messages and reducing the number of RREQ messages sent. The improved algorithm prevents low-energy nodes from forwarding RREQ messages, so that data packets avoid low-energy nodes during transmission, thereby prolonging the survival time of lowenergy nodes. The simulation results show that the survival rate of nodes in the improved algorithm is higher than that of the original algorithm, which prolongs the life cycle of nodes, delays the appearance of network segmentation, and further extends the life cycle of the entire network. The improved routing algorithm is more suitable for the monitoring and management system of nonheritage tourist attractions, effectively ensuring the monitoring range of the system.

4.3. Time Delay Analysis of Reader RF Circuit. Considering that the delay of a single-stage circuit should be in the order of hundreds of ps and the consistency is very good, the delay caused by the random fluctuation of the circuit should be smaller. Compared with the time delay of the ns level, the circuit such as undersampler brings delay fluctuations that are generally small. However, due to the obvious electromagnetic effect and skin effect of PCB microstrip lines in high-speed circuits, the parameters of the transmission line have obvious effects on signal delay and distortion. At the same time, the matching requirements of the transmission line in the radio frequency circuit are very high; otherwise, it will cause the reflected waveform due to the mismatch, which will affect the delay of the AM signal. Therefore, this research ignores the delay effect of the undersampling circuit and takes the transmission delay caused by the PCB microstrip line in the radio frequency circuit as the focus of the analysis. Combined with Mobile GIS-related protocols, it mainly analyzes the signal strength attenuation and delay of uniform microstrip lines in the working frequency bands of $915 \mathrm{MHz}$ and $2.4 \mathrm{GHz}$ suitable for indoor positioning. Combined with the equivalent analysis circuit of the uniform microstrip line, the simulation test is performed on the ADS platform and the influence of the frequency band, line length, and line width is analyzed. The analysis result of the parameter influence is shown in Figure 3. The initial microstrip line width is $0.5 \mathrm{~mm}$, the line length is $40 \mathrm{~mm}$, and the dielectric substrate thickness is $0.3 \mathrm{~mm}$. It can be seen from Figure 3 that, for the microstrip line transmitter in the $915 \mathrm{MHz}$ frequency band, the attenuation is relatively small, with a delay of $0.2442 \mathrm{~ns}$, while the $2.4 \mathrm{GHz}$ frequency band is approximately without attenuation, with a delay of $0.2446 \mathrm{~ns}$. In the $800 \mathrm{MHz}$ frequency band, the transmission line medium appears resonance, which causes the transmission loss to fluctuate, but the delay variation varies little with frequency. From Figure 3, it can be seen that the transmission coefficient attenuation increases with the increase of the microstrip line 


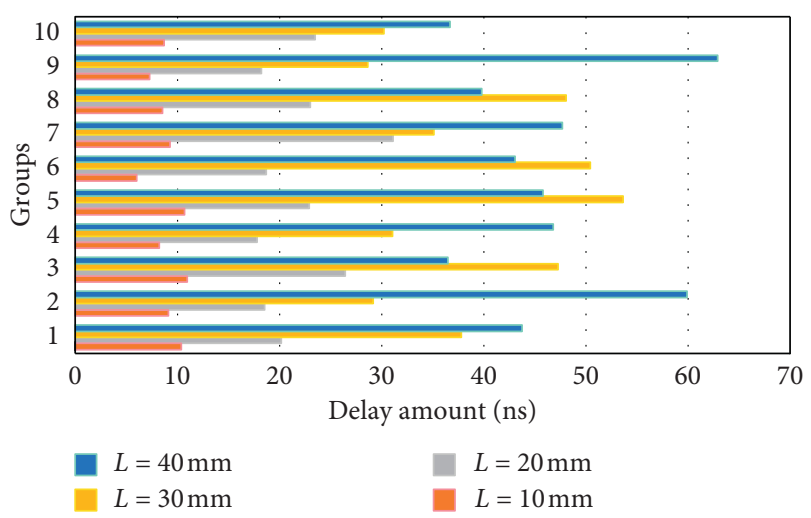

FIGURE 3: Result of analysis of the influence of parameters.

width, and the oscillation of the same period appears; the delay amount also shows the peak oscillation of the same period with the increase of the line width; the larger the width, the higher the peak; and the maximum it is about $5 \mathrm{~ns}$ (line width is $40 \mathrm{~mm}$ ). Comparative analysis shows that the delay oscillation period corresponding to different line widths is the same as the oscillation period of the transmission coefficient, indicating that the oscillation period has nothing to do with the line width and the signal frequency. Therefore, in this study, it is assumed that the line width is a constant value (about $0.5 \mathrm{~mm}$ ), and the influence of line width changes on the transmission delay is ignored. It can be seen from Figure 3 that the transmission coefficient decreases with the increase of the line length; that is, the attenuation increases. The delay increases obviously with the increase of the line length, and the maximum is $3 \mathrm{~ns}$ (the line length is $0.5 \mathrm{~mm}$ ). Therefore, when analyzing the Mobile GIS system implementation circuit, if the line length is considered comprehensively, its length can generally reach $0.2 \mathrm{~m}$ and above, and the delay will be several $\mathrm{ns}$, which will cause positioning errors that cannot be ignored.

4.4. Analysis of Performance Test Results. The simulation data is generated strictly in accordance with the information exchange format designed by the system. The acquired simulation data set includes three categories: terminal location data, terminal real-time location query data, and terminal historical location query data. In the simulation data set, the information interaction data between the PC client and the terminal is not included. The reason is that removing the information interaction data between the PC client and the terminal can simplify the test process. The information interaction data is time-consuming in information processing compared to terminal query the data is low. In the performance test, replacing the interactive data between the PC client and the terminal with terminal query data actually makes the test conditions more stringent. Performance verification is divided into two aspects: terminal access capability test; query performance test. The terminal access capability test is mainly determined by whether the location data is processed in time and correctly. The performance test results are shown in Figure 4. Send the

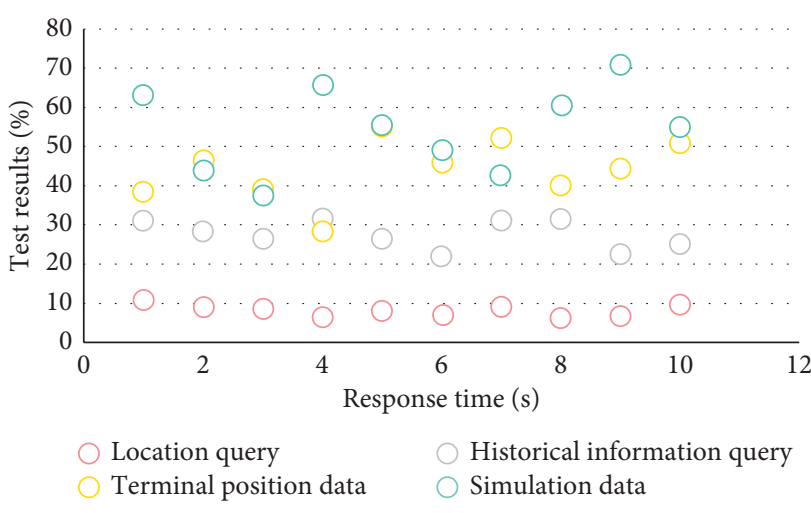

FIgure 4: Performance test results.

simulated location data to the server software. For real-time location data, check whether the real-time data processing meets the design requirements through the database storage records. If the sent real-time location information can be stored smoothly within 1 minute, the location data processing performance is considered to meet the design requirements. For the query request data, it is verified by the following methods: in the simulation query data transmission process, the handheld management terminal is used to send real-time location query and historical location query, and the query result is obtained according to whether the handheld management terminal query response time is within the specified time ( 3 seconds) to determine whether the query response time can meet the design requirements. In terms of location data processing capabilities, the server software can initially store 5,000 records per second in the database. When the amount of data increases, the data insertion speed per second stabilizes at more than 2,000 records. In terminal query, the average response time of realtime location query is $2 \mathrm{~s}$. The response time of historical location query is affected by data insertion, and the initial response time is about $3 \mathrm{~s}$ on average. According to the test results, the server software can meet the design needs of 50,000 terminals. The query response time can be less than $3 \mathrm{~s}$ in real-time location query. Historical location query is affected by data insertion, and the response time will increase with the amount of data. The initial response time is less than $3 \mathrm{~s}$. After 5 days of data accumulation, the response time is about $5 \mathrm{~s}$. In this system, the terminal reports realtime location information to the communication and information format conversion module according to the specified frequency, and the communication and information format conversion module transfers the terminal realtime location information to the location service platform, which is stored by the location service platform, and the scenic spot management platform passes. The location service platform obtains real-time location information of the terminal. Due to the difference in the use of the terminal, the frequency of the terminal reporting location information is not the same. For example, under normal circumstances, in actual use, due to the fast running speed of the vehicle, in order to better capture the change of the vehicle position, the frequency of reporting the position of the vehicle terminal is 
usually set within 10 seconds. For other terminals, it is captured and judged according to the behavior of the terminal user. The main location information report frequency is usually 1 minute. In special cases, the terminal location reference frequency may be modified as required. In order to ensure that the GPU can receive the latest location information for each terminal, the period for the GPU to receive real-time terminal location information from the GPU must match its frequency location information reporting terminal, so the graphical point management platform receives real-time information of the terminal from the location service platform period, and location information also varies with different types of terminals. For example, for in-vehicle terminals, the period for the graphical management platform to receive location information from the location service platform is also less than 10 seconds, and for other terminals, the period may be 1 minute.

\subsection{Implementation Effect of the Monitoring and Management} System Intangible Heritage Tourist Attractions. When tourists are waiting for sightseeing vehicles at the station, the node location service terminal reports the current location of the tourists to the location service platform by reading the information of the tourists' Mobile GIS card. The daily management of the scenic spot is to use the system and various management terminals to realize personnel and vehicle management, dispatch service mode, and monitor the scenic environment. Figure 5 shows the implementation effect of the monitoring and management system of intangible heritage tourist attractions. The scenic area management module can obtain tourist distribution, travel team location, vehicle location, location management personnel location, and other pieces of information from the location service platform. Through the analysis and processing of the scenic area management platform, it can use handheld management intelligent navigation service terminals and vehicle-mounted intelligent navigation services. The terminal can complete the real-time dispatch and command operation of the vehicles, tourist teams, and management personnel in the scenic area; in addition, it can also monitor the environment in the scenic area. The staff of the scenic spot will equip the handheld intelligent search and rescue terminal for the self-service tourists in the scenic spot, which can realize a one-key alarm in the event of accidents such as falls. After receiving the alarm information, the command center automatically generated rescue plans based on the location information of the rescuers and quickly formed a search and rescue team to go to the rescue location to start search and rescue. Using Beidou positioning to realize intelligent navigation to search and rescue points and through $3 \mathrm{G}$ mobile communication, Beidou short messages continuously report location information to the command center to ensure that the command center grasps the rescue situation in real time and achieves efficient search and rescue for rescuers. Through the implementation of the monitoring and management system, tourist attractions can optimize the company's marketing, sales, and tourist services and other related business processes and realize the automated

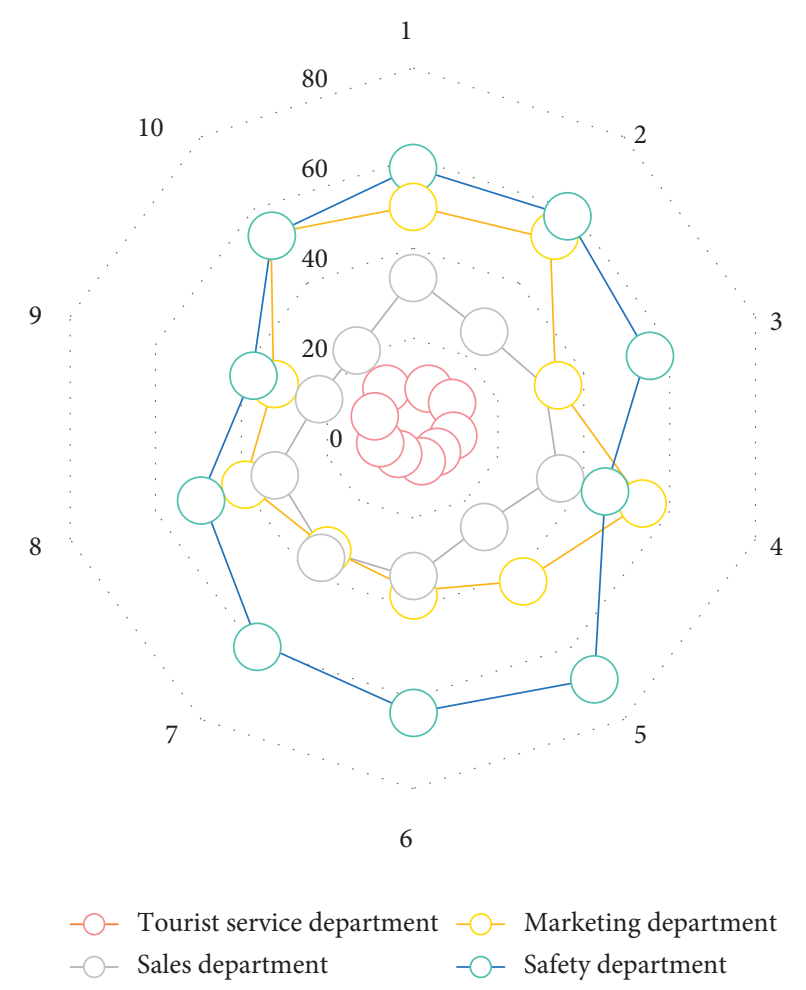

FIGURE 5: Implementation effect of scenic spot monitoring and management system.

operation of various businesses, avoiding various disadvantages that may arise from traditional manual operations and improving the staff of relevant departments. Realize reasonable market segmentation and tourist segmentation through statistical analysis of market information, timely discover potential market opportunities and potential tourists, determine the focus of marketing promotion, and adopt differentiated marketing and sales strategies for different markets and different tourists. The implementation of the tourist attraction monitoring management system can realize the sharing of tourist information within the scope of the panoramic area. The marketing department, sales department, and tourist service department of the scenic spot can query relevant tourist information in real time and quickly according to their own needs and explore the potential needs of tourists in all aspects and improve the quality of tourism services in various departments. Based on the analysis of the development status of local tourist attractions, this research improves the traditional tourist relationship management model centered on channel tourists, proposes a new tourist relationship management model centered on channel tourists and consumer tourists, and emphasizes that the management of channel tourists and consumer tourists should be carried out at the same time, promote each other, jointly improve and achieve a win-win situation among tourist attractions, consumer tourists, and channel tourists, and provide certain guidance and reference for other local tourist attractions in the same industry, to promote the improvement of tourist relationship management and informatization in the tourism industry. The successful 
implementation of the monitoring and management system of tourist attractions can drive the revitalization and development of the regional economy to a certain extent. Our city is one of the first batches of China's best tourist cities as determined by the National Tourism Administration. The construction of tourism projects has made rapid progress in recent years. As the number of tourists in tourist attractions continues to increase, higher requirements are put forward for the management of tourist attractions in our city. The proposal and smooth implementation of this system will help promote the overall informatization construction of tourism service enterprises. It has a strong demonstration and promotion effect in this industry and this field and will play an important role in promoting the vigorous development of tourism in our city.

\section{Conclusion}

In the rapid development of the informatization of the tourism industry, the research of intelligence has been paid more and more attention by experts and scholars. How to improve the planning ability of tourists in the travel process, predict the level of prediction, and provide automatic and convenient tour information has always been the focus of research in this field. The development of today's mobile smart terminal equipment and the continuous evolution of "Internet +" have broken the tourism industry. Developing bottlenecks and improving service quality provide new opportunities.

This paper submits the job requirements of the Internet of Things location system, as well as the internal and external background and current state of development of Wireless Sensor Networks, and then analyzes the important role of wireless sensor placement and summarize some of the shortcomings to existing positioning algorithms, which also make research placement the need for is clearer. It explains some theoretical knowledge related to positioning, analyzes and compares many popular positioning systems or algorithms, and highlights the advantages and disadvantages of positioning systems or algorithms. Then the shorter path algorithm was improved and a better placement scheme was obtained.

As the concept of ecological protection gradually gains popularity among the people, the protection of intangible cultural heritage tourist attractions has received extensive attention from all walks of life. With the development and progress of science and technology, the Internet of Things has developed rapidly in the past ten years and has been gradually applied to various fields. This research applies the Internet of Things technology to the monitoring and management of intangible heritage tourist attractions, uses the technical characteristics of the Internet of Things to monitor and manage the Internet of Things, and proposes a set of effective solutions to develop intangible cultural heritage based on the Internet of Things. Monitoring and management system for tourist attractions. According to the current actual business needs, combined with the expected development goals, the tourism scenic spot monitoring management system brings a new business operation method to the tourism scenic spot monitoring management through advanced technology and finally realizes a set of management control as the core and tourist management as the main line.

\section{Data Availability}

No data were used to support this study.

\section{Conflicts of Interest}

The authors declare that they have no conflicts of interest.

\section{References}

[1] Z. Zhou, B. Chen, and H. Yu, "Understanding RFID counting protocols," IEEE/ACM Transactions on Networking, vol. 24, no. 1, pp. 312-327, 2016.

[2] L. Wang, T. Gu, X. Tao, and J. Lu, "Toward a wearable RFID system for real-time activity recognition using radio patterns," IEEE Transactions on Mobile Computing, vol. 16, no. 1, pp. 228-242, 2017.

[3] A. Tewari and B. B. Gupta, "Cryptanalysis of a novel ultralightweight mutual authentication protocol for IoT devices using RFID tags," Journal of Supercomputing, vol. 73, no. 3, pp. 1-18, 2017.

[4] X. Liu, B. Xiao, K. Li et al., "RFID estimation with blocker tags," IEEE/ACM Transactions on Networking, vol. 25, no. 1, pp. 224-237, 2017.

[5] L. Shangguan, Z. Yang, A. X. Liu et al., "STPP: spatial-temporal phase profiling-based method for relative RFID tag localization," IEEE/ACM Transactions on Networking, vol. 25, no. 1, pp. 1-14, 2017.

[6] R. Y. Zhong, S. Lan, C. Xu et al., "Visualization of RFIDenabled shopfloor logistics big data in cloud manufacturing," The International Journal of Advanced Manufacturing Technology, vol. 84, no. 1-4, pp. 5-16, 2016.

[7] W. Luo, Y. Qiao, S. Chen, and M. Chen, "An efficient protocol for RFID multigroup threshold-based classification based on sampling and logical bitmap," IEEE/ACM Transactions on Networking, vol. 24, no. 1, pp. 397-407, 2016.

[8] N. Kumar, K. Kaur, S. C. Misra, and R. Iqbal, "An intelligent RFID-enabled authentication scheme for healthcare applications in vehicular mobile cloud," Peer-to-Peer Networking and Applications, vol. 9, no. 5, pp. 824-840, 2016.

[9] D. Patron, W. Mongan, T. P. Kurzweg et al., "On the use of knitted antennas and inductively coupled RFID tags for wearable applications," IEEE Transactions on Biomedical Circuits and Systems, vol. 10, no. 6, pp. 1047-1057, 2016.

[10] M. Mayer and N. Goertz, "RFID tag acquisition via compressed sensing: fixed vs. random signature assignment," IEEE Transactions on Wireless Communications, vol. 15, no. 3, pp. 2118-2129, 2016.

[11] L. Yan, C. Di, Q. Wu et al., "Sequential fusion estimation for multisensor systems with non-Gaussian noises," Science China Information Sciences, vol. 63, no. 12, pp. 1-13, 2020.

[12] W.-T. Chen, "Optimal frame length analysis and an efficient anti-collision algorithm with early adjustment of frame length for RFID systems," IEEE Transactions on Vehicular Technology, vol. 65, no. 5, pp. 3342-3348, 2016.

[13] Y. Yin, L. Xie, J. Wu, and S. Lu, "Focus and shoot: exploring auto-focus in RFID tag identification towards a specified area," IEEE Transactions on Computers, vol. 65, no. 3, pp. 888-901, 2016. 
[14] A. Motamedi, M. M. Soltani, S. Setayeshgar, and A. Hammad, "Extending IFC to incorporate information of RFID tags attached to building elements," Advanced Engineering Informatics, vol. 30, no. 1, pp. 39-53, 2016.

[15] M. Mohandes, M. Deriche, H. Ahmadi, M. Kousa, and A. Balghonaim, "An intelligent system for vehicle access control using RFID and ALPR technologies," Arabian Journal for Science and Engineering, vol. 41, no. 9, pp. 3521-3530, 2016.

[16] K.-H. Wang, C.-M. Chen, W. Fang, and T.-Y. Wu, "On the security of a new ultra-lightweight authentication protocol in IoT environment for RFID tags," The Journal of Supercomputing, vol. 74, no. 1, pp. 65-70, 2018.

[17] M. B. de Morais Franca, F. J. O. Morais, P. Carvalhaes-Dias, L. C. Duarte, and J. A. Siqueira Dias, "A multiprobe heat pulse sensor for soil moisture measurement based on PCB technology," IEEE Transactions on Instrumentation and Measurement, vol. 68, no. 2, pp. 606-613, 2019.

[18] M. Z. Rafique, M. N. Ab Rahman, N. Saibani, N. Arsad, and W. Saadat, "RFID impacts on barriers affecting lean manufacturing," Industrial Management \& Data Systems, vol. 116, no. 8, pp. 1585-1616, 2016.

[19] R. Malekian, A. F. Kavishe, B. T. Maharaj, P. K. Gupta, G. Singh, and H. Waschefort, "Smart vehicle navigation system using hidden markov model and RFID technology," Wireless Personal Communications, vol. 90, no. 4, pp. 17171742, 2016.

[20] D. Jayawardana, S. Kharkovsky, R. Liyanapathirana, and $\mathrm{X}$. Zhu, "Measurement system with accelerometer integrated RFID tag for infrastructure health monitoring," IEEE Transactions on Instrumentation and Measurement, vol. 65, no. 5, pp. 1163-1171, 2016.

[21] Y. Bendavid and M. Bourgault, "A living laboratory for managing the front-end phase of innovation adoption: the case of RFID implementation," International Journal of Project Organisation \& Management, vol. 2, no. 1, pp. 129136, 2017.

[22] W. Tounsi, N. Cuppens-Boulahia, F. Cuppens, and G. Pujolle, "Access and privacy control enforcement in RFID middleware systems: proposal and implementation on the fosstrak platform," World Wide Web, vol. 19, no. 1, pp. 41-68, 2016.

[23] S. Mondal, D. Kumar, and P. Chahal, "A continuous-mode single-antenna harmonic RFID tag," IEEE Microwave and Wireless Components Letters, vol. 30, no. 4, pp. 441-444, 2020.

[24] T. Noor, A. Habib, Y. Amin, J. Loo, and H. Tenhunen, "Highdensity chipless RFID tag for temperature sensing," Electronics Letters, vol. 52, no. 8, pp. 620-622, 2016.

[25] M. Borgese, F. A. Dicandia, F. Costa, S. Genovesi, and G. Manara, "An inkjet printed chipless RFID sensor for wireless humidity monitoring," IEEE Sensors Journal, vol. 17, no. 15, pp. 4699-4707, 2017.

[26] A. Wickramasinghe, D. C. Ranasinghe, C. Fumeaux, K. D. Hill, and R. Visvanathan, "Sequence learning with passive RFID sensors for real-time bed-egress recognition in older people," IEEE Journal of Biomedical and Health Informatics, vol. 21, no. 4, pp. 917-929, 2017.

[27] B.-H. Liu, N.-T. Nguyen, V.-T. Pham, and Y.-H. Yeh, “A maximum-weight-independent-set-based algorithm for reader-coverage collision avoidance arrangement in RFID networks," IEEE Sensors Journal, vol. 16, no. 5, pp. 1342-1350, 2016.

[28] D. Betancourt, K. Haase, A. Hübler, and F. Ellinger, "Bending and folding effect study of flexible fully printed and late-stage codified octagonal chipless RFID tags," IEEE Transactions on Antennas and Propagation, vol. 64, no. 7, pp. 2815-2823, 2016. 